\title{
Les mégaprojets à Montréal, de Drapeau à Coderre
}

\author{
Pierre-Mathieu Le Bel et Salvador David Hernandez ${ }^{1}$
}

\section{INTRODUCTION}

Les mégaprojets urbains (MPU) sont à la source de nombreux conflits. Ces conflits pour le contrôle du territoire, sont liés à la logique capital-logement, mais ils ne se développent pas dans le vide, ils sont intriqués à des conflits de plus grande ampleur, liés au système politique et à l'héritage historique du lieu.

Les mégaprojets urbains (MPU) sont à la source de nombreux conflits. Ces conflits pour le contrôle du territoire, sont liés à la logique capital-logement, mais ils ne se développent pas dans le vide, ils sont intriqués à des conflits de plus grande ampleur, liés au système politique et à l'héritage historique du lieu. Les MPU sont des composantes importantes dans la restructuration du rôle de l'instance municipale et le contexte actuel de restructuration du capitalisme à l'échelle mondiale.

Montréal participe à cette dynamique tout en ayant des modalités propres. L'administration publique de Montréal a, par exemple, une grande dépendance fiscale vis-à-vis des impôts fonciers et un caractère néo-corporatiste (Hamel 2006; Montpetit 2003). Ce caractère implique l'établissement de relations étroites entre les acteurs économiques, particulièrement immobiliers, et la ville. Cette alliance a été particulièrement forte durant les périodes d'effervescence des MPU, soit quand les prix de l'immobilier augmentent et avec eux, les investissements dans ce secteur. L'administration Jean Drapeau (1954-1957 et 1960-1986) et celle de Gérald Tremblay (20022012) constituent deux périodes de transformations urbaines importantes où les MPU étaient au centre de la stratégie de développement urbain. La première est une période de mise en place d'une politique de type "urban renewal » et la seconde de mise en marche de politiques néo-libérales. Ces deux périodes ont modifié la gestion publique et ont transformé les actions collectives en augmentant l'opposition et la contestation face aux MPU.

\section{MÉGAPROJETS URBAINS SOUS LE RÈGNE DE JEAN DRAPEAU}

\section{Les décennies 1960 et 1970 ont été riches en MPU de facture fonctionnaliste, particulièrement en Amérique du Nord. La ville de Montréal - alors métropole du Canada - ne faisait pas exception.}

Les décennies 1960 et 1970 ont été riches en MPU de facture fonctionnaliste, particulièrement en Amérique du Nord. La ville de Montréal - alors métropole du Canada - ne faisait pas exception. S'inspirant de la Charte d'Athènes et de la vision de Le Corbusier, c'est dans un esprit fonctionnaliste et « hygiéniste » pour reprendre un terme qui rappelle Hausmann, que l'administration Drapeau a mené à terme de nombreux MPU qui ont modifié le paysage de la ville. Les discours légitimant de ces projets les dépeignaient comme une entreprise d'éradication des « taudis ». D'importants quartiers populaires se sont vus rasés en tout (Goose Village), presque entièrement (Redlight, Griffintown) ou en partie (Faubourg à M'lasse) dans une logique que Henri Lefebvre ${ }^{2}$ décrirait comme s'appuyant sur un espace abstrait. La planification de la ville reposait à la fois sur une division fonctionnelle entre espaces de travail, de loisir et de résidence, mais également sur un rôle de premier plan accordé à la circulation automobile. Les MPU de cette époque se sont déployés de telle sorte que la mixité des fonctions était impossible ou fortement découragée.

«Le Québec de cette époque donne l'impression d'être à la fois un vaste chantier en construction et un laboratoire vivant où prennent place une myriade d'expériences et 
d'initiatives [...] la tension entre, d'un côté, la mise en place d'un État centralisateur et, de l'autre, les mouvements sociaux [exigeaient] une participation plus active aux décisions $»^{3}$.

$\mathrm{Au}$ niveau institutionnel, la transformation et la modernisation de l'État ont impliqué des changements importants dans la réglementation et la planification territoriale du développement provincial et municipal. La loi sur les fusions volontaires de 1965 puis la création de la CUM, en 1970, ont été le résultat de la pression du gouvernement provincial pour centraliser les administrations municipales et la planification territoriale. Les pressions d'une économie en restructuration et des groupes économiques ont favorisé aussi la réorientation de la politique municipale impliquant l'établissement d'une relation directe et de caractère corporatif entre quelques-uns des groupes économiques et les décideurs de la ville en matière économique et de planification du développement. À Montréal, ce pacte entre élites a impliqué une présence plus forte des élites économiques dans la vie institutionnelle de la ville, particulièrement de la Chambre de commerce et des acteurs liés au développement immobilier. Cela se traduit par une présence accrue des intérêts des groupes économiques dans les décisions et la gestion municipales. Cela est visible dans la mise en marche des commissions sectorielles, qui déterminent les politiques de développement, formées de représentants du secteur privé et du secteur public. Ces commissions ont coordonné les actions municipales afin d'accélérer et orienter le développement économique dans des secteurs spécifiques. Enfin, elles sont le cadre de négociations ayant lieu à huit clos entre entrepreneurs et décideurs politiques et où se prennent des décisions de planification territoriale soutenues par le pouvoir discrétionnaire du maire et du conseil exécutif.

Les pressions d'une économie en restructuration et des groupes économiques ont favorisé aussi la réorientation de la politique municipale impliquant

l'établissement d'une relation directe et de caractère corporatif entre quelques-uns des groupes économiques et les décideurs de la ville en matière économique et de planification du développement.
Sur le plan économique, à partir des années 1970, la Ville de Montréal a commencé à être significativement affectée par un processus de désindustrialisation ${ }^{4}$. C'est aussi le moment où Toronto est devenue la métropole économique du Canada. La crise économique de 1973, de même que l'expérience olympique financièrement et architecturalement douteuse marquèrent un point tournant dans la réalisation des MPU. La première mit fin à des projets presque mégalomanes comme celui de la cité Concordia, la seconde fut un trauma encore évoqué aujourd'hui pour refroidir les ardeurs des promoteurs et politiciens.

Du côté de la protestation, les MPU ont généré des changements sur les communautés affectées au plan de l'organisation de la protestation citoyenne et encouragé le développement de mouvements sociaux. Pendant cette période, la question de l'accès au logement, mais surtout la lutte contre l'expropriation - comme dans Milton Park occupent l'avant-scène. La contestation face aux MPU et surtout face aux démolitions a été à la base de la création du Front d'action politique (FRAP) au niveau municipal. En citant Manuel Castells ${ }^{5}$, un mouvement de « coordination d'expériences » s'est construit en instrumentalisant le contexte d'opposition au MPU. Face à l'administration Drapeau, qualifiée par certains d'autocratique et de centralisée, la venue du Rassemblement des Citoyens de Montréal (RCM) a alors été présentée comme une alternative politique incarnant, sous la forme d'une coalition, les intérêts des mouvements civiques, syndicaux et sociaux qui s'opposaient à l'administration de Drapeau. Les propositions de décentralisation et de consultation publique mises de l'avant par l'équipe Doré ont modifié la procédure d'implantation des MPU dans les communautés. Doré a institutionnalisé la tenue d'assemblées publiques de consultation face aux MPU entre 1988 et 1994.

Pourtant, l'idéal incarné par ces assemblées et le discours municipal de transformation de la gouvernance se sont rapidement inscrits en contradiction avec les pratiques politiques du Rassemblement des Citoyens de Montréal basées sur la centralisation de la prise de décisions aux mains du comité exécutif. Concrètement, des projets comme ceux de l'îlot Overdale et de la carrière Miron vont finir par diviser la coalition et les factions les plus progressistes se retireront. 


\section{MONTRÉAL À L'ÈRE DES MÉGAPROJETS CONTEMPORAINS}

Qu'est-ce qui distingue la période actuelle de celle des années 1960? Les MPU transforment la métropole à travers plusieurs dimensions : 1) La dimension urbanistique: Les mégaprojets urbains imposent de nouveaux patrons de rue, une restauration complète, voire un remplacement, des infrastructures d'égouts, d'énergie, de télécommunication. En outre, non seulement le mégaprojet dicte à l'ensemble urbain une certaine orientation, mais les intérêts des promoteurs sont susceptibles d'orienter en amont la forme urbanistique des MPU; 2) La dimension économique : les MPU sont censés représenter une source de revenu pour l'ensemble de l'agglomération. On vantera l'activité générée par sa construction et les emplois futurs. Le sous-entendu étant que si l'espace commercial est disponible, des entreprises s'y établiront; 3) La dimension sociale et culturelle; ici, le discours veut, par exemple, que le mérite architectural des mégaprojets serve d'impulsion au tourisme international. Nombre de chercheurs qui se sont intéressés aux mégaprojets comme composantes de la stratégie de développement urbain, ont mis en lumière le rôle du « branding $»^{6}$. Cette dimension, de par son caractère presque omniprésent, constitue une nouveauté au sens où elle se pose comme caractéristique d'un urbanisme postmoderne ${ }^{7}$. Il va sans dire que tous ne tablent pas autant sur les mêmes dimensions, entre le musée Gugenheim de Bilbao, les London Docklands ou la reconstruction du World Trade Center de New York, la variété des modalités est très grande, même si tous ces projets se déploient sur l'ensemble de ces dimensions. L'instrumentalisation de la dimension symbolique ainsi que le passage d'un État planificateur à un État médiateur au niveau local incarnent l'innovation des MPU contemporains.

Les projets de développement urbain sont l'expression la plus évidente de la Nouvelle politique urbaine pour qui l'intervention étatique doit être dirigée vers la construction d'infrastructures urbaines destinées à accélérer la circulation des capitaux, la promotion de l'entrepreneuriat et les Partenariats Public-Privé.
La planification urbaine s'est orientée vers l'attraction d'investisseurs et de capitaux sur des territoires où la ville tente d'offrir un avantage comparatif aux investisseurs. Les projets de développement urbain sont l'expression la plus évidente de la Nouvelle politique urbaine pour qui l'intervention étatique doit être dirigée vers la construction d'infrastructures urbaines destinées à accélérer la circulation des capitaux, la promotion de l'entrepreneuriat et les Partenariats PublicPrivé $^{8}$. Le manque de propositions alternatives et de participation réelle des communautés affectées dans les MPU est justifié juridiquement par le besoin de confidentialité pendant le processus de sélection des contractants. L'état d'exception caractérise également les MPU contemporains et a une triple nature'. Il s'agit d'abord d'une exception de planification visible par la mise en place des Plans particuliers d'urbanisme qui viennent modifier les normes et visions des plans antérieurs. C'est, ensuite, une exception de gouvernance qu'on remarque par l'usage plus fréquent du droit de véto de certains élus, ainsi que par l'intégration d'acteurs non-élus au processus décisionnel. C'est finalement une exception juridique caractérisée par la création de lois ad hoc qui peut aller jusqu'à l'application d'une forme d'état d'urgence coercitif sur un territoire déterminé.

C'est après une pause d'environ 25 ans que Montréal a recommencé à miser sur les MPU. Pierre Bourque arrive au pouvoir en 1994 sous la bannière de Vision Montréal et avec une nouvelle proposition de centralisation du pouvoir : "une île, une ville". Sous son mandat, les consultations publiques pour les projets sont abandonnées à cause de leur coût monétaire, mais également pour leur effet sur la dynamique des affaires ${ }^{10}$. Sous son administration, la réforme municipale de 2001 a été associée à l'application des principes de la Nouvelle gestion publique sur le territoire métropolitain et elle a forcé la fusion des villes situées sur l'île de Montréal.

Garant d'une plus grande autonomie aux arrondissements de la ville en partie défusionnée, l'administration Tremblay va donner une importance particulière à la participation citoyenne. En 2002, elle réalisera un exercice de planification 
participative qui orientera par la suite l'administration, le Sommet de Montréal. Cette administration amplifiera le mouvement de décentralisation et introduira des modifications importantes dans la planification de MPU, principalement avec le retour à la politique de consultation publique et la mise en marche d'un plan de planification de MPU à long terme appelé Montréal 2025. Ce plan a pour objectif de faciliter l'arrivée des investisseurs et du capital dans la ville par l'entremise de la mise en marche de dispositifs et stimuli i.e. exemptions en matière d'impôts. En 2005, la Ville a adopté une stratégie de soutien aux grands projets urbains devant être mis en œuvre sur une période de 20 ans $^{11}$ visant à attirer des capitaux au niveau international et à construire des infrastructures reliées à l'exploitation de ces avantages comparatifs dans le contexte de la concurrence internationale. Le plan d'urbanisme adopté en 2004 confère également un rôle important à la planification par la voie de grands projets ${ }^{12}$. Ces MPU sont étroitement liés aux intérêts des capitaux locaux et internationaux et au renouvellement de la gestion publique, qui deviendrait plus orientée vers le marché. Par exemple, le Groupe d'intervention stratégique et tactique a été mis en place dans le but explicite de commander des études et de soutenir les promoteurs dans leurs démarches.

\section{Le plan d'urbanisme adopté en 2004 confère également un rôle important à la planification par la voie de grands projets. Ces MPU sont étroitement liés aux intérêts des capitaux locaux et internationaux et au renouvellement de la gestion publique, qui deviendrait plus orientée vers le marché.}

En guise d'exemple de MPU importants à Montréal, le Quartier des spectacles (QDS) repré-

\section{CONCLUSION}

Ces exemples de MPU montréalais montrent comment l'événementiel joue maintenant un rôle important. Si Montréal vise explicitement à faire mousser son image de métropole culturelle, les mégaprojets urbains tendent à joindre les éléments symboliques à leur stratégie. Ce faisant, les investisseurs viennent non pas seulement chercher le profit provenant d'avantages comparatifs liés à sente un investissement majeur - plusieurs centaines de millions de dollars entre fonds publics et privés - de même qu'un développement économique axé sur la culture, il constitue le symbole d'un branding urbain cohérent avec les tendances de la postmodernité et est stratégique dans la mesure où Montréal mise sur ces forces vis-à-vis de la ville reine. Outre le QDS, le travail rassemblant instances municipale et provinciale autour du site olympique et de l'Espace pour la vie misent eux aussi sur l'événementiel et le symbolique.

On n'oubliera pas les mégaprojets hospitaliers du CUSM et du CHUM. Bien que l'événementiel ne fasse pas partie intégrante de ses deux projets, il importe d'en souligner des aspects symboliques qui légitiment leur réalisation. D'abord, l'importance du secteur de la santé dans les priorités sociales des québécois et de la recherche médicale pour les investisseurs. Ensuite, le fait qu'il y ait un hôpital anglophone et un hôpital francophone.

Parallèlement, la protestation en réponse à la mise en place de grands projets et face aux processus de gentrification dans les quartiers centraux a augmenté. Parmi les grands projets qui ont entraîné de fortes mobilisations de la part des groupes citoyens et communautaires, nous pouvons mentionner le déménagement du casino dans le quartier Pointe Saint-Charles, Benny Farm à NDG, le projet Matrec de transbordement de déchets à SaintHenri, le réaménagement du canal Lachine et le projet de l'échangeur Turcot. On notera que les MPU du domaine des transports comme ce dernier ou l'élargissement du boulevard Notre-Dame sont ceux qui attirent le plus de contestations tout en étant ceux mobilisant le moins l'événementiel ou le culturel.

une localisation strictement cartésienne (davantage le fait des MPU des années 1960 et 1970), mais plutôt également extraire une valeur d'un patrimoine intangible, situé dans le bien commun des manières de faire propres aux lieux.

L'événementiel est rentable pour les promoteurs, mais donne cependant une prise aux groupes 
d'opposants. Ainsi, le symbole misant sur un substrat qui fait partie du bien commun est, alors, susceptible de légitimer l'action des décideurs et des promoteurs, mais aussi de servir de base à la contestation en vertu de cette appartenance au bien commun. Cela expose encore une autre tendance des MPU contemporains, tant à Montréal qu'ailleurs, celle de coopter un minimum de groupes de la société civile afin de légitimer les MPU. Ainsi, le CUSM signe une entente de principe avec des groupes communautaires choisis, la Régie des installations olympiques (RIO) invite des groupes communautaires à des consultations sur son avenir et le QDS rassemble des partenaires tant commerciaux que communautaires. On peut soit s'en réjouir en y voyant une opportunité de concertation bénéfique à tous, soit s'en désolé en constatant le graduel amincissement des espaces de contestation.

\section{BIBLIOGRAPHIE ET NOTES}

${ }^{1}$ Les auteurs sont respectivement chargé de cours en géographie à l'Université Concordia et candidat au doctorat en Études urbaines et touristiques de l'UQAM.

${ }^{2}$ Voir LEFEBVRE, H. (2000). La production de l'espace, Paris, Economica.

${ }^{3}$ Voir HÉTU, L. (2009). Une ville sous tutelle : brève histoire de l'administration de Montréal, Montréal, Liber.

${ }^{4}$ Voir LEVINE, M. (1991). The reconquest of Montreal. Philadelphie, Temple University Press.

${ }^{5}$ Voir CASTELLS, M. (1973). Luttes urbaines. Paris

: François Maspéro.

${ }^{6}$ Voir ROSEMBERG, 2000.

${ }^{7}$ Voir HARVEY, D. (1989). The condition of postmodernity. Oxford, Blackwell. et SOJA, E. (2000). Postmetropolis, New York, Blackwell.

${ }^{8}$ Voir SWYNGEDOUW, E., F. MOULAERT et RODRIGUEZ, A. (2002). « Neoliberal Urbanization in Europe: Large-Scale Urban Development Projects and the New Urban Policy », Antipode, vol. 34, $\mathrm{n}^{\circ} 3$, p. 542-577.

${ }^{9}$ Voir AGAMBEN, G. (2005) State of exception, Chicago, University of Chicago Press.

${ }^{10}$ Voir HAMEL, P. (2006). Développement local et action communautaire à l'heure $d u$ néocorporatisme. Chicoutimi : J.-M. Tremblay. En ligne <http://dx.doi.org/doi:10.1522/000210418>. Consulté le 2 décembre 2013.

${ }^{11}$ Voir VILLE DE MONTRÉAL (2005). ImaginerRéaliser Montréal 2025. Un monde de créativité et de possibilité, Montréal, Service de la mise en valeur du territoire et du patrimoine, Direction de la planification stratégique.

${ }^{12}$ Voir DUCAS, S. (2010). «La gestion de grands projets urbaines à Montréal ». Urbanité, vol. Hiver 2010, p. 1. 


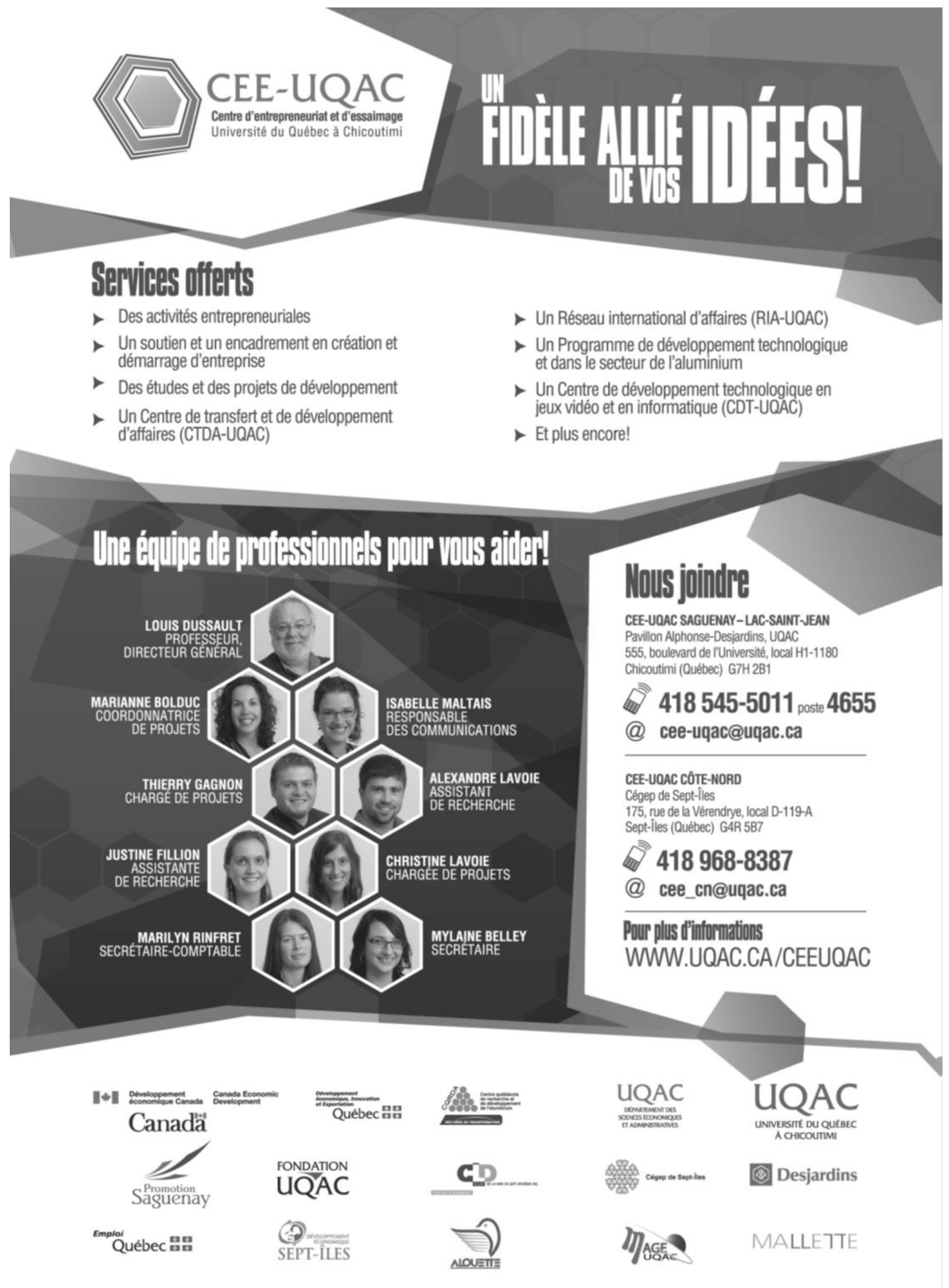

